\title{
Tropical Cyclone Genesis Guidance Using the Early Stage Dvorak Analysis and Global Ensembles
}

\author{
MUNEHIKO YAMAGUCHI \\ Meteorological Research Institute, Japan Meteorological Agency, Tsukuba, Japan \\ NAOHISA KOIDE \\ Japan Meteorological Agency, Tokyo, Japan
}

(Manuscript received 27 April 2017, in final form 3 October 2017)

\begin{abstract}
TC genesis guidance using the early stage Dvorak analysis technique (EDA) and global ensembles is investigated as one of the statistical-dynamical TC genesis guidance schemes. The EDA is a scheme that enables the analysis of tropical disturbances at earlier stages by adding T numbers of 0.0 and 0.5 to the conventional Dvorak technique. This unique analysis method has been in operation at JMA since 2001. The global ensembles used in this study are the ECMWF, JMA, NCEP, and UKMO ensembles covering from 2010 to 2013. First, probabilities that tropical disturbances analyzed with the EDA reach tropical storm intensity within a certain lead time up to 5 days are statistically investigated. For example, the probabilities that a tropical disturbance analyzed with T numbers of $0.0,0.5$, and 1.0 reaches tropical storm intensity within 2 days are $15 \%, 23 \%$, and $57 \%$, respectively. While the false alarm ratio (FAR) is found to decrease if the global ensembles simulate the tropical disturbance analyzed with the EDA in the models, it tends to decrease with the increasing number of such ensemble members. Also, it should be noted that the probability of detection (POD) decreases with the increasing number of such ensemble members. One of the potential uses of these verification results is that forecasters could issue TC genesis forecasts by counting ensemble members that successfully simulate a targeted tropical disturbance and then refer to the FAR and POD corresponding to the number of the ensemble members. These would provide some confidence information of the forecasts.
\end{abstract}

\section{Introduction}

Tropical cyclone (TC) genesis is one of the key forecast challenges for many of the operational TC forecasting centers around the world. Forecasting the location and timing of TC genesis accurately is important from a perspective of effective early warnings for disasters associated with TCs. According to Fudeyasu et al. (2014), among all TCs that made landfall over Japan, $14 \%$ of them made landfall within 2 days from TC genesis while the percentage is $44 \%$ for the Philippines. Accurate TC genesis forecasting is indispensable for such areas in particular, as the time from TC genesis to landfall is relatively short.

Landsea (2014) reviewed the current status of operational TC genesis forecasting at the Eighth World Meteorological Organization (WMO) International

Corresponding author: Munehiko Yamaguchi, myamagu@ mri-jma.go.jp
Workshop on Tropical Cyclones (IWTC) in 2014. Many regional specialized meteorological centers (RSMCs) and tropical cyclone warning centers (TCWCs) provide TC genesis products up to 5 days in advance for the public. While TC track forecasts heavily rely on the outputs of numerical weather prediction (NWP) models, TC genesis forecasts depend more on subjective analyses and decisions by forecasters. As such, there is increasing demand from the operational to the research communities for the development of objective tools to help forecasters make TC genesis forecasts. Currently, there seem to be three main approaches to the development for TC genesis forecast tools: statistical, NWP model based, and statistical-dynamical. One of the examples of the statistical approach is TC genesis guidance using the Dvorak technique (Dvorak 1975, 1984). Cossuth et al. (2013) statistically calculated the likelihood that tropical disturbances analyzed using the Dvorak technique at the National Hurricane Center (NHC) and the Central Pacific Hurricane Center from 
2001 to 2011 reached TC intensity. The results can be used as a baseline or climatology for disturbance-based TC genesis forecasts. For the feasibility of NWP models for TC genesis forecasts, Elsberry et al. (2009) evaluated four deterministic global models during the Tropical Cyclone Structure (TCS08) and The Observing System Research and Predictability Experiment (THORPEX) Pacific Asian Regional Campaign (T-PARC) and showed that a consensus of the track forecasts to 3 days provided good guidance for the pre-tropical cyclone seedings. Halperin et al. (2013, 2017) evaluated five deterministic global models and demonstrated that they have become a more reliable source of TC genesis guidance. Majumdar and Torn (2014) examined probabilistic TC genesis forecasts using single-model-based ensembles and showed the potential of the ensembles for probabilistic TC genesis forecasts out to 5 days, and Yamaguchi et al. (2015) demonstrated the benefits of combining multiple ensembles (i.e., multimodel grand ensemble) for TC activity (genesis and subsequent track) forecasts. Elsberry et al. $(2010,2011)$ evaluated the feasibility of the ECMWF 1-month ensemble for an extended range of TC genesis forecasts. For a statistical-dynamical approach, Dunion (2013) developed a disturbancefollowing TC genesis index (TCGI) for identifying the $0-48$ - and $0-120$-h probability of TC genesis and reported the effectiveness of TCGI using the Brier skill score as a verification metric.

The RSMC Tokyo-Typhoon Center issues TC advisories for developing tropical disturbances. Advisories are issued when the disturbances are expected to reach tropical storm intensity, which has maximum sustained surface winds (10-min average) of $34 \mathrm{kt}$ or more (where $1 \mathrm{kt}=0.51 \mathrm{~m} \mathrm{~s}^{-1}$ ), within $24 \mathrm{~h}$. Other than this, the RSMC Tokyo-Typhoon Center does not provide TC genesisrelated products to the public. Meanwhile, a unique TC analysis technique called early stage Dvorak analysis (EDA) was developed and has been in operation since 2001 (Tsuchiya et al. 2001; Kishimoto et al. 2007; Kishimoto 2009). In a conventional Dvorak techniquebased TC intensity estimate, a tropical number (or T number) is assigned to a storm, and this $\mathrm{T}$ number is linked to TC intensity. The T number is classified into 15 categories ranging from 1.0 to 8.0 with a 0.5 interval. The larger the $\mathrm{T}$ number is, the stronger the tropical disturbances/cyclones are. In general, TCs of tropical storm intensity or greater have a T number of 2.5 or more (Koba et al. 1990; Dvorak 1984). The EDA is a scheme that makes it possible to analyze tropical disturbances in their earlier stages by adding $T$ numbers of 0.0 and 0.5 to the conventional classification of the Dvorak technique.

In this study, the likelihood that tropical disturbances analyzed with the EDA at the RSMC Tokyo-Typhoon
Center reach tropical storm intensity up to 5 days is calculated. This work can be seen as an extension of Cossuth et al. (2013), but this study uses the EDA-based T numbers instead of the conventional Dvorak technique-based $\mathrm{T}$ numbers. Second, we examine how this likelihood changes if the disturbance analyzed with the EDA is successfully simulated by global ensembles. The ensemble forecasts used in this study are the global ensembles from the European Centre for Medium-Range Weather Forecasts (ECMWF), the JMA, the National Centers for Environmental Prediction (NCEP), and the Met Office in the United Kingdom (UKMO). The purpose of this study is to provide a climatological likelihood that tropical disturbances in the western North Pacific that are so weak that they are not analyzed by the conventional Dvorak technique reach tropical storm intensity and to examine the feasibility of TC genesis guidance using the EDA and global ensembles as one of the statistical-dynamical approaches.

This paper is organized as follows. Section 2 describes data and methodology. Section 3 describes the results of verification. Section 4 is a summary of this study.

\section{Data and methodology}

\section{a. General methodology}

The cumulative probabilities that a tropical disturbance analyzed with the EDA in the western North Pacific basin will reach tropical storm intensity are calculated as a function of time from the EDA's analysis time to 5 days ahead. These probabilities are calculated for $T$ numbers of $0.0,0.5$, and 1.0 , respectively. Note that the $\mathrm{T}$ number assigned to a tropical disturbance can change as it develops or weakens and that each analysis is handled independently in the verification. This verification provides a probability that a tropical disturbance analyzed with a $\mathrm{T}$ number of $0.0,0.5$, or 1.0 reaches tropical storm intensity within $X$ days from the analysis time. Using a contingency table shown in Table 1, this probability is written as $a /(a+b)$. Note that $c$ and $d$ are both zero. One minus this probability, $b /(a+b)$, can be seen as a baseline for the false alarm ratio (FAR; Wilks 2011) of the $X$-day TC genesis forecasts.

Next, using global ensembles, we examine how the FAR changes as a function of the number of ensemble members that successfully simulate the tropical disturbance in the models from the EDA's analysis time to $X$ days ahead. In addition to the FAR, probability of detection (POD) is also evaluated (see section 3 for more details). In this study, we verify 2 - and 5-day TC genesis forecasts (i.e., $X=2$ or 5).

The best-track data used in this study are taken from the RSMC Tokyo-Typhoon Center's best-track dataset. 
TABLE 1. Contingency table, where $a$ is the number of cases where the event (i.e., TC genesis) was correctly forecast to occur; $b$ is the number of cases where the event was forecast, but it did not occur; $c$ is the number of cases where the event occurred despite not being forecast; and $d$ is the number of cases where the event did not occur after a forecast that it would not occur.

\begin{tabular}{llcc}
\hline \hline & & \multicolumn{2}{c}{ Observed } \\
\cline { 3 - 4 } & & Yes & No \\
\hline Forecast & Yes & $a$ & $b$ \\
& No & $c$ & $d$ \\
\hline
\end{tabular}

This dataset includes the location and timing that tropical disturbances reach tropical storm intensity, which is a threshold for TC genesis in this study, as well as the maximum sustained wind of tropical disturbances/ cyclones. Note that the maximum sustained winds in the archive are 10-min-average winds.

\section{b. Early stage Dvorak analysis}

JMA has operated the EDA (Tsuchiya et al. 2001) since 2001. In the conventional Dvorak analysis, tropical disturbances/cyclones are assigned T numbers of 1.0-8.0 with a 0.5 interval depending on their strength. Meanwhile, the EDA analyzes tropical disturbances whose strength has yet to reach a $T$ number of 1.0 based on the following objective conditions:

1) A convective cloud system has persisted for $12 \mathrm{~h}$ or more.

2) The cloud system has a cloud system center (CSC) defined within a diameter of $2.5^{\circ}$ latitude or less.

3) The CSC has persisted for $6 \mathrm{~h}$ or more.

4) The cloud system has an area that is dense and cold $\left(-31^{\circ} \mathrm{C}\right.$ or colder) and that appears less than $2^{\circ}$ latitude from the center.

5) The above overcast is more than $1.5^{\circ}$ latitude in diameter.

When three out of the five conditions are met, a T number of 0.0 is assigned, while a $\mathrm{T}$ number of 0.5 is assigned when four out of the five conditions are met. Note that a $T$ number of 1.0 is also assigned with the EDA when all five conditions are satisfied. These criteria are exactly the same as those used for a $\mathrm{T}$ number of 1.0 in the conventional Dvorak analysis. In operations at JMA, in general, the EDA is succeeded with the conventional Dvorak analysis when an analyzed tropical disturbance further develops to a $\mathrm{T}$ number of 1.5 or more.

All cases analyzed with the EDA from 2010 to 2013 are verified in this study. Table 2 shows the number of TCs, the storms analyzed with EDA, and the times when the storm is analyzed with a $\mathrm{T}$ number of $0.0,0.5$, or 1.0 in 2010-13.
TABLE 2. The number of TCs, storms analyzed with EDA, and numbers of times when the storm is analyzed with a $\mathrm{T}$ number of $0.0,0.5$, or 1.0 during $2010-13$.

\begin{tabular}{lrrrr}
\hline \hline & 2010 & 2011 & 2012 & 2013 \\
\hline $\begin{array}{l}\text { No. of TCs } \\
\text { No. of storms analyzed with EDA }\end{array}$ & 14 & 21 & 25 & 31 \\
$\quad \begin{array}{l}\text { No. of analysis times with a } \\
\quad \text { T number of 0.0 }\end{array}$ & 196 & 167 & 138 & 150 \\
$\quad$ No. of analysis times with a & 147 & 133 & 117 & 88 \\
$\quad$ T number of 0.5 & & & & \\
$\quad \begin{array}{l}\text { No. of analysis times with a } \\
\quad \text { T number of 1.0 }\end{array}$ & 52 & 50 & 28 & 48 \\
& & & & \\
\hline
\end{tabular}

\section{c. Global ensembles}

Global ensembles of the ECMWF, JMA, NCEP, and UKMO from 2010 to 2013 are used in this study (see Table 3). These data are available from the THORPEX International Grand Global Ensemble (TIGGE; Swinbank et al.2016). Note that only the 1200 UTC initial times for the forecasts are used. The tropical disturbances and TCs in the models are tracked using the methodology described in Vitart et al. (2012), where the maximum vorticity at $850 \mathrm{hPa}$ with a warm-core structure (a local maximum of averaged temperature between 500 and $200 \mathrm{hPa}$ is defined as the center of the warm core, and from this center location, the temperature must decrease by at least $0.5^{\circ} \mathrm{C}$ in all directions within a distance of $8^{\circ}$ latitude) is tracked every $6 \mathrm{~h}$ and both minimum sea level pressure and a maximum wind speed are detected within a distance of $2^{\circ}$ latitude from the location of the vorticity local maximum.

While the EDA is conducted four times a day (0000, 0600, 1200, and 1800 UTC) at most, storm tracking data are available once a day in this study (1200 UTC initials only). For example, when verifying the likelihood that a tropical disturbance analyzed with the EDA at 0000 UTC reaches tropical storm intensity within 2 days, 12 and $60-\mathrm{h}$ forecasts initiated at 1200 UTC on the previous day are used to see if the ensemble is successful in simulating the targeted storm. Similarly, 18- and 66-h forecasts and 24- and 72-h forecasts are used for the EDA at 0600 and 1200 UTC, respectively. For the EDA at 1800 UTC, the ensemble forecasts initiated at 1200 UTC on the same day could be used, but the ensemble forecasts on the previous day are used in this study in light of the large amount of computational time required for ensemble forecasting and the available time of such ensemble datasets from foreign centers. A threshold distance of $500 \mathrm{~km}$ is applied to determine whether the simulated storm represents a storm analyzed with the EDA. 
TABLE 3. Ensemble configurations for the ECMWF, JMA, NCEP, and UKMO ensembles during the study period from 2010 to 2013.

\begin{tabular}{lcccc}
\hline \hline & $\begin{array}{c}\text { ECMWF } \\
\text { (Europe) }\end{array}$ & $\begin{array}{c}\text { JMA } \\
\text { (Japan) }\end{array}$ & $\begin{array}{c}\text { NCEP } \\
\text { (United States) }\end{array}$ & $\begin{array}{c}\text { UKMO } \\
(\text { United Kingdom) }\end{array}$ \\
\hline $\begin{array}{l}\text { Horizontal resolution of the } \\
\text { model (forecast length) }\end{array}$ & $\begin{array}{c}\text { TL639 (0-10 days), TL319L91 } \\
(10-15 \text { days })\end{array}$ & TL319 (0-days) & $\begin{array}{c}\text { T254 (0-8 days), T190 } \\
(8-16 \text { days })\end{array}$ & $0.55^{\circ} \times 0.88^{\circ}(0-15$ days $)$ \\
$\begin{array}{l}\text { Ensemble size } \\
\begin{array}{l}\text { Horizontal grid spacing in } \\
\text { the TIGGE archive }\end{array}\end{array}$ & $\begin{array}{c}51 \\
21\end{array}$ & 51 & $1.0^{\circ} \times 1.0^{\circ}$ & 24 \\
\hline
\end{tabular}

\section{Results}

\section{a. TC genesis probability based on EDA}

Figure 1 shows cumulative probabilities that a tropical disturbance analyzed with the EDA reaches tropical storm intensity within a certain lead time of up to 5 days. When looking at a certain lead time, the probabilities become larger for larger $\mathrm{T}$ numbers. For example, the probabilities that a tropical disturbance analyzed with $\mathrm{T}$ numbers of $0.0,0.5$, and 1.0 reaches tropical storm intensity within 2 (5) days from the EDA's analysis time are $15 \%, 23 \%$, and $57 \%(30 \%, 36 \%$, and $65 \%)$, respectively. In other words, the FARs of 2- (5) day TC genesis forecasts just based on the EDA for T numbers of $0.0,0.5$, and 1.0 are $85 \%, 77 \%$, and $43 \%(70 \%, 64 \%$, and $35 \%$ ), respectively. These numbers can be seen as a baseline for FARs of 2- and 5-day TC genesis forecasts.

\section{b. TC genesis probability based on EDA and global ensembles}

According to the verification results in Fig. 1, if we issue 2-day TC genesis forecasts based on the T numbers specified with the EDA, the FARs will be $85 \%, 77 \%$, and $43 \%$ for $\mathrm{T}$ numbers of $0.0,0.5$, and 1.0 , respectively. Figure 2 shows the FAR and POD of 2-day TC genesis forecasts based on the EDA's T number and global ensembles. The results are examined for each $T$ number and each global ensemble from ECMWF, JMA, NCEP, and UKMO. The ensemble probability ( $x$ axes in the plots in Fig. 2) is defined as the ratio of the ensemble members in which a targeted tropical disturbance is tracked both at the analysis time of the EDA and at 2 days from the analysis time to the total number of the ensemble members (see Table 3 ). The verification results correspond to lines with closed circles in Fig. 2. The FAR and POD are defined as

$$
\mathrm{FAR}_{n}=\frac{\sum_{i=n}^{N} \mathrm{NO}_{i}}{\sum_{i=n}^{N}\left(O_{i}+\mathrm{NO}_{i}\right)} \text { and }
$$

$$
\mathrm{POD}_{n}=\frac{\sum_{i=n}^{N} O_{i}}{\sum_{i=1}^{N} O_{i}},
$$

where $n$ is bin number and $N$ is the total number of bins. Here, $O$ and NO are the numbers of forecast cases where the event occurred and did not occur, respectively (see Table 4 for more details). The numerator and denominator of FAR correspond to $b$ and $a+b$ in the contingency table shown in Table 1 , respectively. Similarly, the numerator and denominator of POD correspond to $a$ and $a+c$, respectively, in the contingency table shown in Table 1.

For a $\mathrm{T}$ number of 0.0 with the ECMWF ensemble (Fig. 2a), for example, the FAR with an ensemble probability of $0 \%$ is $86 \%$. This is exactly the same as the FAR with the EDA information only in this case the ensemble probability of $0 \%$ means that forecasts are issued regardless of the ensemble information. If half or more of the ensemble members (i.e., the ensemble probability of $50 \%$ ) meet the conditions

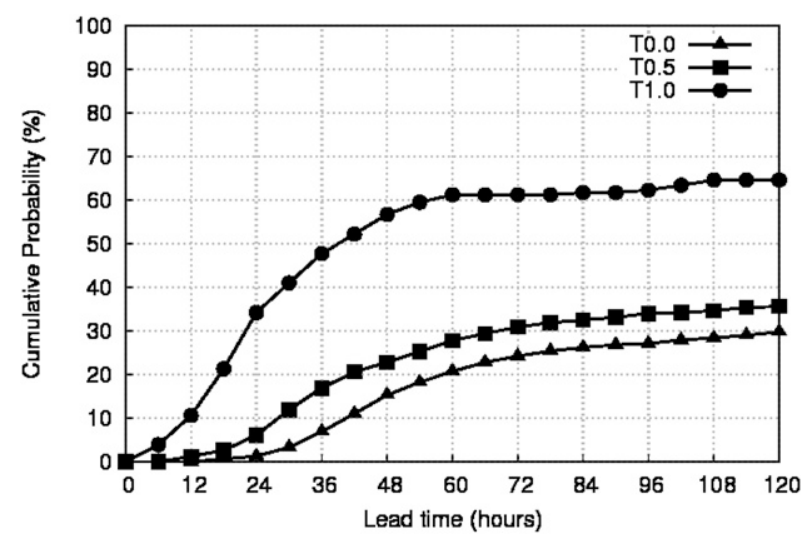

FIG. 1. Cumulative probabilities that a tropical disturbance analyzed with the EDA reaches tropical storm intensity within a certain lead time are shown along the $x$ axis. The triangles, squares, and circles in the lines are for verification of $\mathrm{T}$ numbers of $0.0,0.5$, and 1.0 , respectively. 
(a)

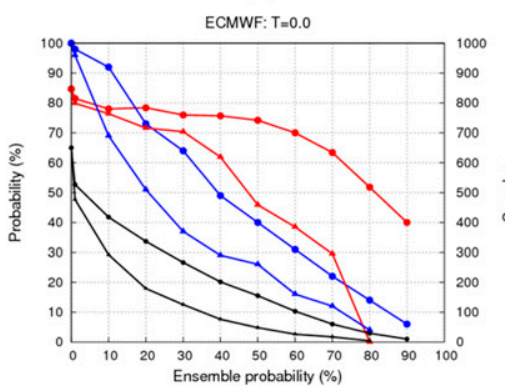

(d)

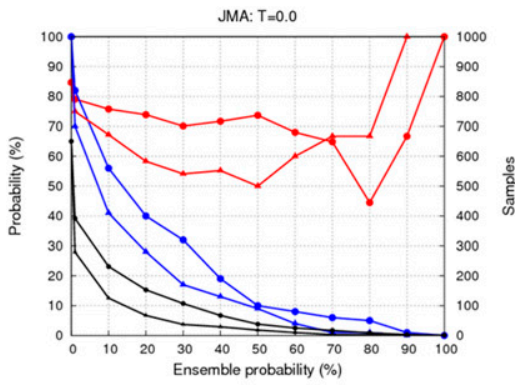

(g)

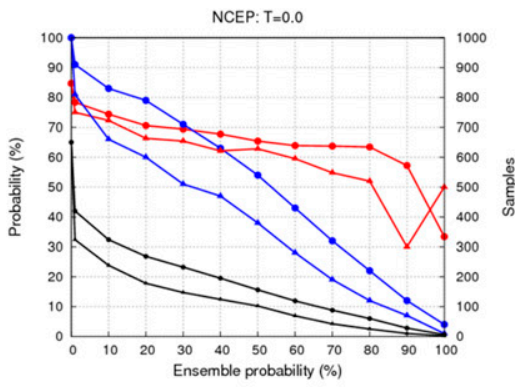

(j)

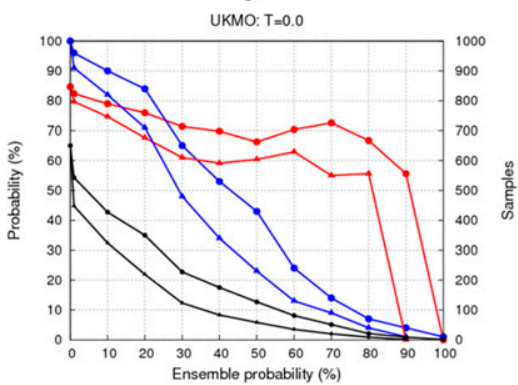

(b)

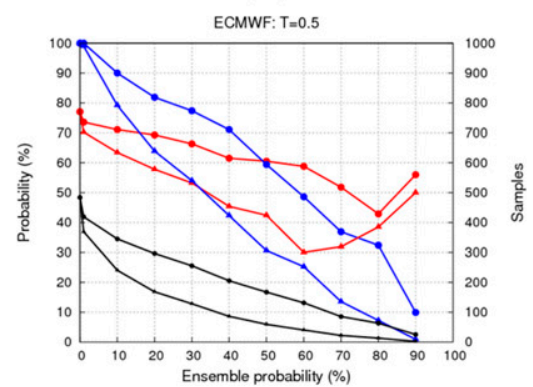

(e)

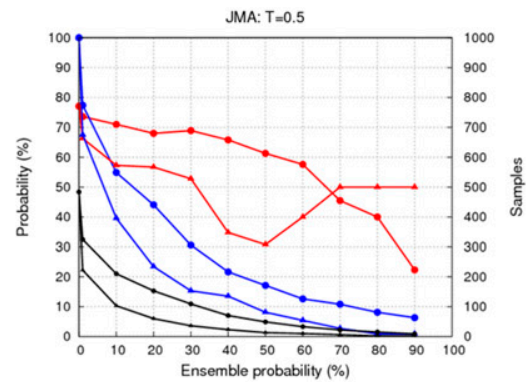

(h)

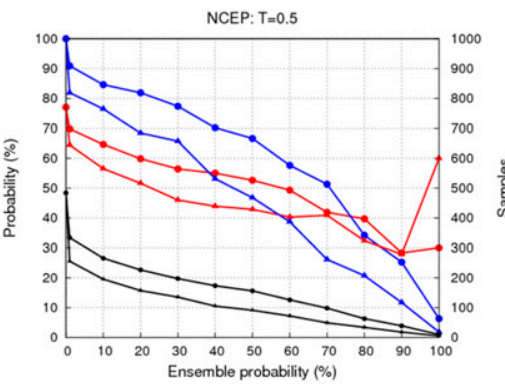

(k)

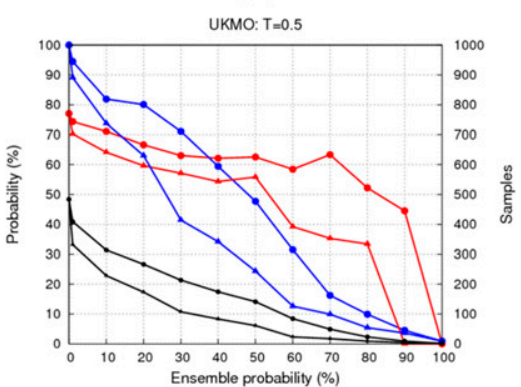

(c)

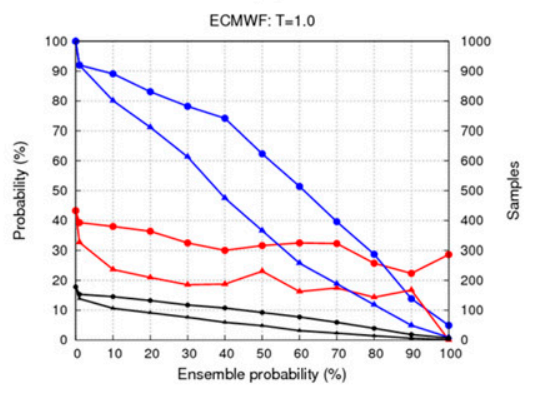

(f)

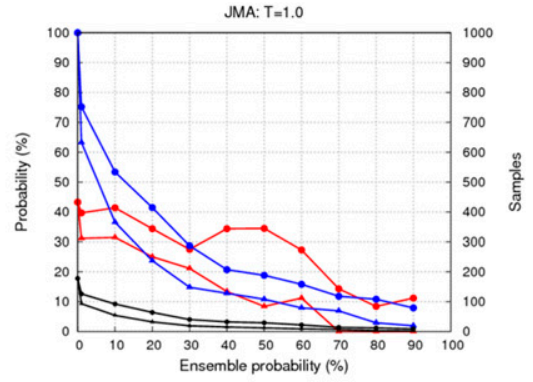

(i)

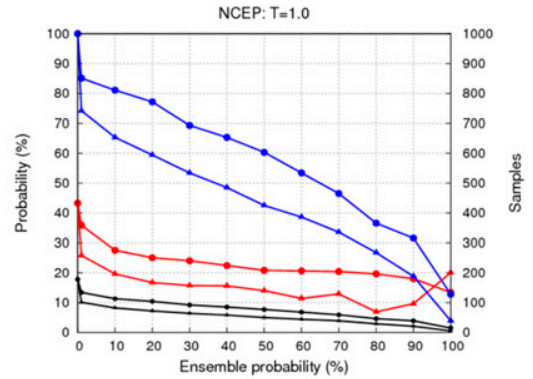

(l)

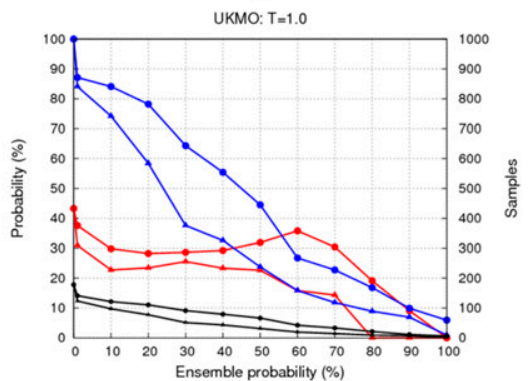

FIG. 2. FAR (red) and POD (blue) of 2-day TC genesis forecasts for the (a)-(c) ECMWF, (d)-(f) JMA, (g)-(i) NCEP, and (j)-(1) UKMO ensembles, corresponding to the $y$ axis on the left. Verification results for T numbers (left) 0.0, (center) 0.5, and (right) 1.0. The circles and triangles in the lines are for different conditions used to calculate ensemble probabilities along the $x$ axis (see text). Black lines are the number of times that an event (TC genesis) is forecast to occur, corresponding to the $y$ axis on the right.

mentioned above, the FAR becomes $75 \%$, which is an $11 \%$ decrease compared to forecasts without the ensemble forecasts. If we focus on the ensemble probability of $50 \%$, the largest decrease in the FAR is seen in the NCEP ensemble, where the FAR values drop from $89 \%$ to $65 \%$, from $77 \%$ to $53 \%$, and from $43 \%$ to $21 \%$ for $\mathrm{T}$ numbers of $0.0,0.5$, and 1.0 , respectively. 
TABLE 4. Probabilistic contingency table used in this study.

\begin{tabular}{cccc}
\hline \hline Bin No. & $\begin{array}{c}\text { Forecast } \\
\text { probabilities }(\%)\end{array}$ & $\begin{array}{c}\text { Observed } \\
\text { occurrences }\end{array}$ & $\begin{array}{c}\text { Observed } \\
\text { nonoccurrences }\end{array}$ \\
\hline 1 & $P_{1}=0$ & $O_{1}$ & $\mathrm{NO}_{1}$ \\
2 & $0<P_{2}<10$ & $O_{2}$ & $\mathrm{NO}_{2}$ \\
3 & $10 \leq P_{3}<20$ & $O_{3}$ & $\mathrm{NO}_{3}$ \\
4 & $20 \leq P_{4}<30$ & $O_{4}$ & $\mathrm{NO}_{4}$ \\
5 & $30 \leq P_{5}<40$ & $O_{5}$ & $\mathrm{NO}_{5}$ \\
6 & $40 \leq P_{6}<50$ & $O_{6}$ & $\mathrm{NO}_{6}$ \\
7 & $50 \leq P_{7}<60$ & $O_{7}$ & $\mathrm{NO}_{7}$ \\
8 & $60 \leq P_{8}<70$ & $O_{8}$ & $\mathrm{NO}_{8}$ \\
9 & $70 \leq P_{9}<80$ & $O_{9}$ & $\mathrm{NO}_{9}$ \\
10 & $80 \leq P_{10}<90$ & $O_{10}$ & $\mathrm{NO}_{10}$ \\
11 & $90 \leq P_{11}<100$ & $O_{11}$ & $\mathrm{NO}_{11}$ \\
12 & $P_{12}=100$ & $O_{12}$ & $\mathrm{NO}_{12}$ \\
\hline
\end{tabular}

In general, the FAR becomes smaller with increasing ensemble probability. The decrease in the FAR is encouraging, but it should be noted that this is achieved at the cost of the reduction in the POD. For example, NCEP's 2-day forecast PODs with an ensemble probability of $50 \%$ are $54 \%, 67 \%$, and $60 \%$ for T numbers of $0.0,0.5$, and 1.0 , respectively. Figure 2 can be used by forecasters to determine the confidence level of their forecasts in view of the FAR and POD. One of the potential uses of the verification results shown in Fig. 2 is that forecasters could issue 2-day TC genesis forecasts with high confidence when the FAR is expected to be smaller than a certain percentage, say $30 \%$. It should be noted that setting this percentage lower reduces the number of genesis forecasts issued. To secure a certain amount of forecast cases, forecasters could issue the forecasts with medium confidence when the POD is expected to be larger than a certain percentage (e.g., $50 \%)$.

\section{c. Redefining the ensemble probability by adding an additional condition}

The ensemble probability is redefined in the following manner. A condition that the maximum sustained surface wind of the targeted tropical disturbance in the model has to be larger at day 2 than that at the EDA's analysis time is added to the original conditions. The verification results correspond to lines with closed triangles in Fig. 2. In general, the FAR decreases with this new condition compared to that with the original conditions. Forecasters can take advantage of this information to increase the level of confidence of their forecasts.

\section{d. 5-day TC genesis forecasts}

We apply the same verification method when evaluating 5-day TC genesis forecasts. Unlike with the verification for the 2-day TC genesis forecasts shown in
Fig. 2, TC tracking results at day 5 from the EDA's analysis time are used when calculating the ensemble probability. Figure 3 shows the verification results for 5-day TC genesis forecasts. The FAR of the 5-day forecasts is smaller than that of the 2-day forecasts though the number of times that an event (e.g., TC genesis) is forecast to occur is also smaller in the 5-day forecasts. These results indicate that forecasters could issue TC genesis forecasts with high confidence even with a 5-day time range though the number of such forecast cases is limited. The difference in the FARs between bins $1\left(P_{1}=0 \%\right)$ and $2\left(0 \%<P_{2}<10 \%\right)$ is larger than that for the 2-day forecast. This means that if any one of the ensemble members simulates the targeted tropical disturbance at day 5 and at the EDA's analysis time, the chance for the disturbance to reach tropical storm intensity within 5 days largely increases. Meanwhile, the POD of the 5-day forecasts tends to be smaller than that of the 2-day forecasts. This is due mainly to the fact that forecasting the timing and location of TC genesis correctly becomes more difficult with a longer forecast lead time. The difficulties with the 5-day TC genesis forecasts can be also seen from the fact that the difference of the POD between bins $1\left(P_{1}=0 \%\right)$ and $2\left(0 \%<P_{2}<10 \%\right)$ is larger during verification for the 5-day forecasts than that for the 2-day forecasts.

Finally, Tables 5 and 6 show a comparison of the FAR and POD results among the four ensembles. As an example, these are the verification results with an ensemble probability of $50 \%$. In general, the NCEP ensemble has smaller FARs and larger PODs for both 2- and 5-day TC genesis forecasts. Meanwhile, the JMA ensemble tends to have smaller PODs and smaller numbers of times that an event is forecast to occur than the other three NWP models. This would be consistent with Yamaguchi et al. (2015), who showed that the frequency of correct forecasts of TC activity (genesis and the subsequent track) decreases with increasing forecast interval.

\section{Summary}

In this study, the likelihood that tropical disturbances in the western North Pacific reach tropical storm intensity (a maximum sustained surface wind speed of $34 \mathrm{kt}$ or higher) within a certain lead time up to 5 days is statistically examined, followed by an investigation as to how this likelihood changes if global ensembles simulate the tropical disturbance in the model. The EDA, which has been in operation in JMA since 2001, is used to detect tropical disturbances. The EDA is a scheme that enables the analysis of tropical disturbances at earlier stages by adding $\mathrm{T}$ numbers of 0.0 and 0.5 to the 
(a)

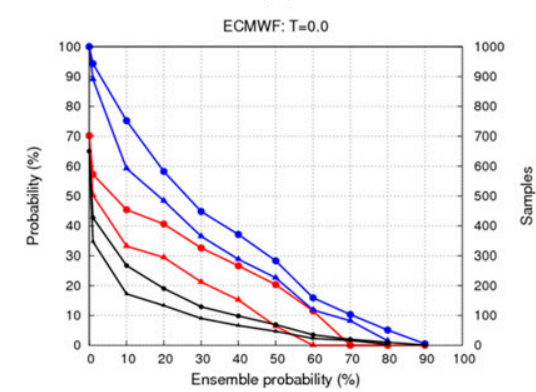

(d)

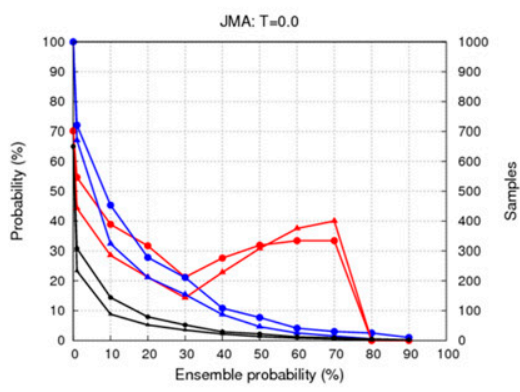

(g)

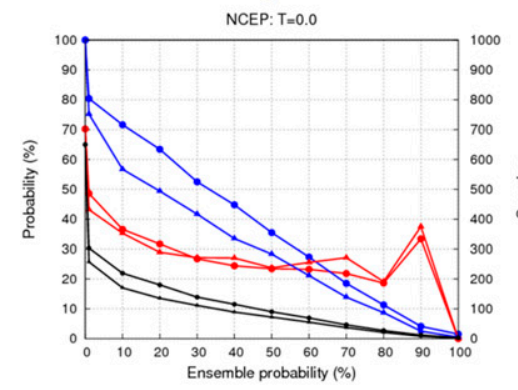

(j)

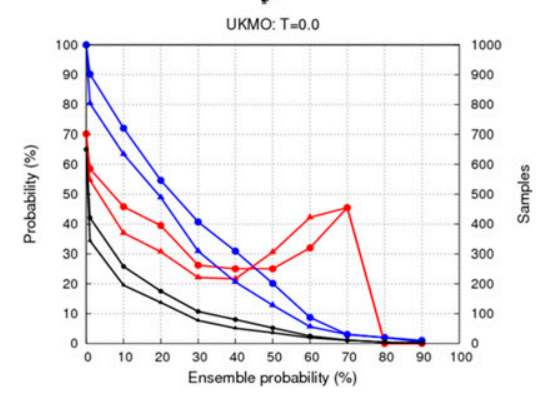

(b)

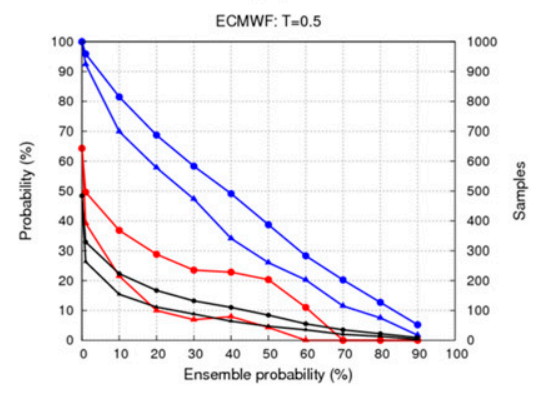

(e)

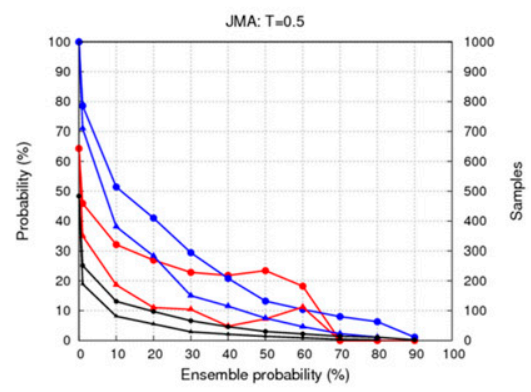

(h)

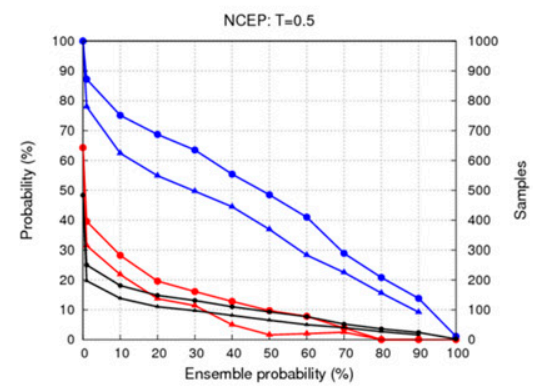

(k)

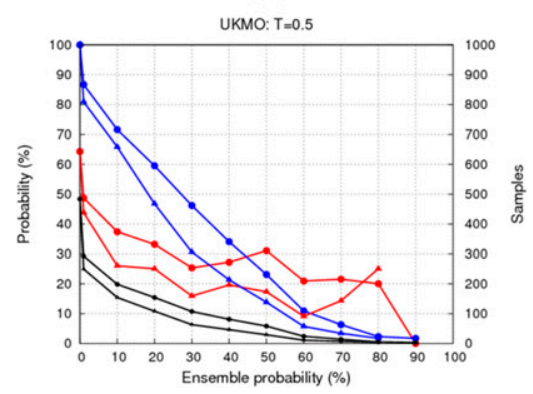

(c)

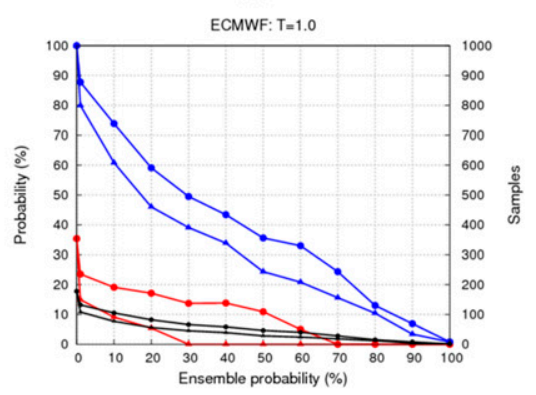

(f)

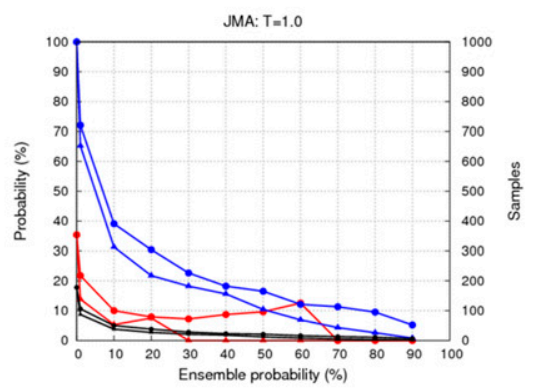

(i)

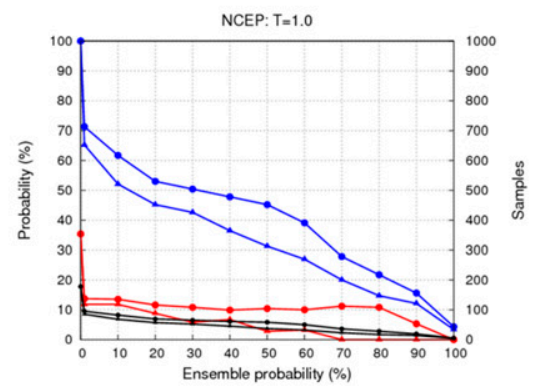

(1)

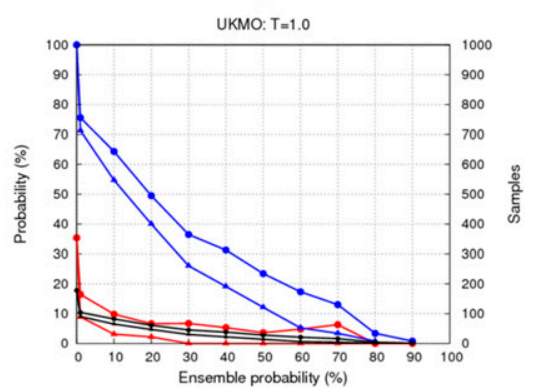

FIG. 3. As in Fig. 2, but for 5-day forecasts.

conventional classification of the Dvorak technique. The global ensembles used in this study are from the ECMWF, JMA, NCEP, and UKMO. The verification period is from 2010 to 2013.

A tropical disturbance analyzed with $\mathrm{T}$ numbers of $0.0,0.5$, and 1.0 reaches tropical storm intensity within 2 (5) days from the EDA's analysis time with probabilities of $15 \%, 23 \%$, and $57 \%(30 \%, 36 \%$, and $65 \%)$, respectively. In other words, the FARs of 2- (5) day TC genesis forecasts just based on the EDA for T numbers of $0.0,0.5$, and 1.0 are $85 \%, 77 \%$, and $43 \%(70 \%, 64 \%$, and $35 \%$ ), respectively. These numbers can be seen as a baseline for the FARs of 2- and 5-day TC genesis forecasts. 
TABLE 5. FAR (\%) among the ECMWF, JMA, NCEP, and UKMO ensembles as well as the EDA-based forecasts. For the ensembles, the FAR results are for verification with an ensemble probability of $50 \%$. Values for the best-performing model are highlighted in boldface.

\begin{tabular}{lccccccr}
\hline \hline & \multicolumn{3}{c}{2 day } & & \multicolumn{3}{c}{5 day } \\
\cline { 2 - 4 } \cline { 6 - 7 } T number & 0.0 & 0.5 & 1.0 & & 0.0 & 0.5 & 1.0 \\
\hline ECMWF & 74 & 60 & 32 & & $\mathbf{2 0}$ & 20 & 11 \\
JMA & 74 & 61 & 34 & & 32 & 23 & 10 \\
NCEP & $\mathbf{6 5}$ & $\mathbf{5 3}$ & $\mathbf{2 1}$ & & 23 & $\mathbf{1 0}$ & 10 \\
UKMO & 66 & 62 & 32 & & 25 & 31 & $\mathbf{3}$ \\
EDA & 85 & 77 & 43 & & 70 & 64 & 35 \\
\hline
\end{tabular}

The FAR is found to decrease if a tropical disturbance analyzed with the EDA is tracked in the global ensembles. In addition, the FAR becomes smaller as the number of ensemble members simulating the disturbance in the model increases. For example, if half or more of the members of the NCEP ensemble simulate the disturbance at the EDA's analysis time and at 2 days from this analysis time, the chances of this disturbance reaching tropical storm intensity within 2 days become $35 \%, 47 \%$, and $79 \%$ for $\mathrm{T}$ numbers of $0.0,0.5$, and 1.0 , respectively. These results are encouraging from an operational forecasting perspective, but it should be noted that the number of successful forecast cases decreases with increasing ensemble probabilities (i.e., the POD decreases with increasing ensemble probabilities by its definition).

One of the potential uses of the verification results in this study is that forecasters could issue 2- or 5-day TC genesis forecasts with confidence information by counting ensemble members that successfully simulate a targeted tropical disturbance and then referring to the FAR and POD corresponding to the ensemble members.

As of February 2016, ECMWF, JMA, and UKMO run their global ensemble twice a day, initiated at 0000 and 1200 UTC, and NCEP runs its ensemble four times a day, at 0000, 0600, 1200, and 1800 UTC. However, the global ensembles used in this study are initiated at 1200 UTC only. As the forecast accuracy is expected to be better with shorter forecast times, making full use of these datasets will further improve the FAR and POD.

TABLE 6. As in Table 5, but for POD (\%).

\begin{tabular}{lrrrrrrr}
\hline & \multicolumn{3}{c}{2 day } & & \multicolumn{3}{c}{5 day } \\
\cline { 2 - 4 } \cline { 6 - 7 } T number & 0.0 & 0.5 & 1.0 & & 0.0 & 0.5 & 1.0 \\
\hline ECMWF & 40 & 59 & $\mathbf{6 2}$ & & 28 & 39 & 36 \\
JMA & 10 & 17 & 19 & 8 & 13 & 17 \\
NCEP & $\mathbf{5 4}$ & $\mathbf{6 7}$ & 60 & & $\mathbf{3 6}$ & $\mathbf{4 9}$ & $\mathbf{4 5}$ \\
UKMO & 43 & 48 & 45 & & 20 & 23 & 23 \\
EDA & 100 & 100 & 100 & & 100 & 100 & 100 \\
\hline
\end{tabular}

Acknowledgments. The authors thank THORPEX International Grand Global Ensemble (TIGGE) for constructing useful and user-friendly portal sites and providing analysis and forecast data of operational ensemble prediction systems. The authors also thank three anonymous reviewers for a thoughtful review and constructive comments. This research was partly supported by the Japan Society for the Promotion of Science KAKENHI (Grant 26282111).

\section{REFERENCES}

Cossuth, J. H., R. D. Knabb, D. P. Brown, and R. E. Hart, 2013: Tropical cyclone formation guidance using pregenesis Dvorak climatology. Part I: Operational forecasting and predictive potential. Wea. Forecasting, 28, 100-118, https://doi.org/ 10.1175/WAF-D-12-00073.1.

Dunion, J., 2013: Development of a probabilistic tropical cyclone genesis prediction scheme. Joint Hurricane Testbed Final Rep., 4 pp., http://www.nhc.noaa.gov/jht/11-13reports/ Final_Dunion_JHT13.pdf.

Dvorak, V. F., 1975: Tropical cyclone intensity analysis and forecasting from satellite imagery. Mon. Wea. Rev., 103, 420-430, https://doi.org/10.1175/1520-0493(1975)103<0420: TCIAAF $>2.0 . \mathrm{CO} ; 2$.

_ 1984: Tropical cyclone intensity analysis using satellite data. NOAA Tech. Rep. NESDIS 11, 47 pp.

Elsberry, R. L., W. M. Clune, and P. A. Harr, 2009: Evaluation of global model early track and formation prediction in the western North Pacific. Asia-Pac. J. Atmos. Sci., 45, 357-374.

- M. S. Jordan, and F. Vitart, 2010: Predictability of tropical cyclone events on intraseasonal timescales with the ECMWF monthly forecast model. Asia-Pac. J. Atmos. Sci., 46, 135-153, https://doi.org/10.1007/s13143-010-0013-4.

,$- \ldots$, and - , 2011: Evaluation of the ECMWF 32-day ensemble predictions during 2009 season of western North Pacific tropical cyclone events on intraseasonal timescales. Asia-Pac. J. Atmos. Sci., 47, 305-318, https://doi.org/10.1007/ s13143-011-0017-8.

Fudeyasu, H., S. Hirose, and H. Yoshioka, 2014: A global view of the landfall characteristics of tropical cyclones. Trop. Cyclone Res. Rev., 3, 178-192.

Halperin, D. J., H. E. Fuelberg, R. E. Hart, J. H. Cossuth, P. Sura, and R. J. Pasch, 2013: An evaluation of tropical cyclone genesis forecasts from global numerical models. Wea. Forecasting, 28, 1423-1445, https://doi.org/10.1175/WAF-D-13-00008.1.

- R. Hart, H. Fuelberg, and J. Cossuth, 2017: The development and evaluation of a statistical-dynamical tropical cyclone genesis guidance tool. Wea. Forecasting, 32, 27-46, https:// doi.org/10.1175/WAF-D-16-0072.1.

Kishimoto, K., 2009: Revision of JMA's early stage Dvorak analysis and its use to analyze tropical cyclones in the early developing stage. RSMC Tokyo-Typhoon Center Technical Review, No. 10, 12 pp., http://www.jma.go.jp/jma/jma-eng/ jma-center/rsmc-hp-pub-eg/techrev/text10-1.pdf.

- T. Nishigaki, S. Nishimura, and Y. Terasaka, 2007: Comparative study on organized convective cloud systems detected through early stage Dvorak analysis and tropical cyclones in early developing stage in the western North Pacific and the South China Sea. RSMC Tokyo-Typhoon Center Tech. Review, No. 9, 14 pp., http://www.jma.go.jp/jma/jma-eng/ jma-center/rsmc-hp-pub-eg/techrev/text9-2.pdf. 
Koba, H., T. Hagiwara, S. Asano, and S. Akashi, 1990: Relationships between CI number from Dvorak's technique and minimum sea level pressure or maximum wind speed of tropical cyclone (in Japanese). J. Meteor. Res, 42, 59-67.

Landsea, C. W., 2014: Cyclogenesis: Operational forecasting perspective. Eighth Int. Workshop on Tropical Cyclones, Jeju, South Korea, WMO/World Weather Research Program, 2.2, https:/www.wmo.int/pages/prog/arep/wwrp/new/documents/ Topic2.2_OperationalForecastingPerspective.pdf.

Majumdar, S., and R. Torn, 2014: Probabilistic verification of global and mesoscale ensemble forecasts of tropical cyclogenesis. Wea. Forecasting, 29, 1181-1198, https://doi.org/ 10.1175/WAF-D-14-00028.1.

Swinbank, R., and Coauthors, 2016: The TIGGE project and its achievements. Bull. Amer. Meteor. Soc., 97, 49-67, https://doi.org/ 10.1175/BAMS-D-13-00191.1.
Tsuchiya, A., T. Mikawa, and A. Kikuchi, 2001: Method of distinguishing between early stage cloud systems that develop into tropical storms and ones that do not. Geophys. Mag., 4, 49-59.

Vitart, F., F. Prates, A. Bonet, and C. Sahin, 2012: New tropical cyclone products on the web. ECMWF Newsletter, No. 130, ECMWF, Reading, United Kingdom, 17-23, https://www. ecmwf.int/sites/default/files/elibrary/2012/17427-new-tropicalcyclone-products-web.pdf.

Wilks, D. S., 2011: Statistical Methods in the Atmospheric Sciences. 3rd ed. Elsevier, 676 pp.

Yamaguchi, M., F. Vitart, S. Lang, L. Magnusson, R. Elsberry, G. Elliott, M. Kyouda, and T. Nakazawa, 2015: Global distribution of the skill of tropical cyclone activity forecasts on short- to medium-range time scales. Wea. Forecasting, 30, 1695-1709, https://doi.org/10.1175/WAF-D-14-00136.1. 